\title{
Relativistic quantum kinetic theory for massive fermions and spin effects
}

\author{
Jian-Hua Gao ${ }^{1}$ and Zuo-Tang Liang ${ }^{2}$ \\ ${ }^{1}$ Shandong Provincial Key Laboratory of Optical Astronomy and Solar-Terrestrial Environment, \\ Institute of Space Sciences, Shandong University, Weihai, Shandong 264209, China \\ ${ }^{2}$ Key Laboratory of Particle Physics and Particle Irradiation (MOE), \\ and Research Center of Particle Science and Technology, Institute of Frontier \\ and Interdisciplinary Science of Shandong University, Qingdao 266237, China
}

(Received 27 February 2019; published 26 September 2019)

\begin{abstract}
We present the complete first order relativistic quantum kinetic theory with spin for massive fermions derived from the Wigner function formalism in a concise form that shows explicitly how the 32 Wigner equations reduce in practice to 4 independent transport equations. We solve the modified on-shell conditions to obtain the general solution and present the corresponding transport equations in three different forms that are suitable for different purposes. We demonstrate how different spin effects arise from the kinetic theory by calculating the chiral separation effect with mass correction, the chiral anomaly from the axial current and the quantum magnetic moment density induced by vorticity and magnetic field. We also show how to generate the global polarization effect due to spin vorticity coupling. The formalism presented may serve as a practical theoretical framework to study different spin effects in relativistic fermion systems encountered in different areas such as heavy ion, astro-particle and condensed matter physics as well.
\end{abstract}

DOI: 10.1103/PhysRevD.100.056021

\section{INTRODUCTION}

Spin plays an essential and fascinating role to probe the underlying structure of theories in different areas of physics. The recent observation [1,2] by STAR Collaboration of the global polarization [3-7] of $\Lambda$ hyperon in noncentral heavy ion collisions opens new directions in the study of hot and dense nuclear matter and motivates particularly further theoretical efforts on the physics of the global polarization effect (GPE) and vorticity [8-17]. What is quite extraordinary in heavy ion collisions is that spin can emerge as a series of macroscopic collective effects such as, besides the GPE [3-7] observed by STAR [1,2], the chiral magnetic effect (CME) [18-20], the chiral vortical effect (CVE), the chiral separation effect (CSE) [21-26] and so on. This is quite different from other high energy reactions and fascinating in its own way. Because the hot and dense system produced in heavy-ion collisions expands and cools down very fast, the natural and promising theoretical framework to deal with these novel collective quantum effects is the relativistic quantum kinetic theory (RQKT).

In recent years there has been a considerable amount of work and significant progresses on the chiral kinetic theory (CKT), i.e., RQKT for massless fermions [26-38]. With the

Published by the American Physical Society under the terms of the Creative Commons Attribution 4.0 International license. Further distribution of this work must maintain attribution to the author(s) and the published article's title, journal citation, and DOI. Funded by SCOAP ${ }^{3}$. running of the beam energy scan program at RHIC and especially the discovery of global polarization at relatively lower energies [1,2], it becomes indispensable to develop a consistent and practical framework of RQKT to be capable of treating various spin effects mentioned above for massive fermions.

The covariant Wigner function formalism is a powerful and systematic quantum kinetic approach [39-43]. It is very successful to derive CKT and to describe CME and CVE consistently. In this massless case, many of the equations decoupled from each other. We note in particular that it has been proved [37] that a disentanglement theorem is valid so that the Wigner equations are reduced to two scalar equations so that it becomes much easier to solve them. However, RQKT for the massive fermions is very different from CKT. Here, in addition to the particle density, the spin polarization vector (SPV) or the axial current will also become a dynamical quantity and the Wigner equations are entangled with each other in much more complicated ways so that transport equations are also much more complicated than those in the chiral limit. So far, the existing works [39-44] on RQKT for massive fermions are restricted to leading order transport equations for SPV, which is enough to deal with the vector current or energy momentum tensor to the first order in $\hbar$. However, they are incapable of accounting for how the GPE can be produced from vorticity and how the chiral anomaly can arise in kinetic theory for massive fermions in a self-contained manner.

The aim of this paper is to derive RQKT with spin for massive Fermions to the first order in $\hbar$ completely and 
study the disentanglement of Wigner equations. We then present the general solution of the modified on-shell conditions at this order. After that, we apply them to various spin effects in relativistic fermion systems to demonstrate how they are derived from RQKT consistently.

The rest of the paper is organized as follow. After this introduction, in Sec. II, we present the Wigner function formalism to the first order in $\hbar$. We show in particular that, by choosing the scalar and axial vector as independent variables, we can sort them in a concise form that shows explicitly all the first order Wigner equations reduce to five transport equations with one subsidiary constraint and five modified on-shell conditions. In Sec. III, we solve the modified on-shell conditions and present the transport equation in three different forms. In Sec. IV, we derive different spin effects from the transport equations and other related equations presented in Secs. II and III. Finally, in Sec. V, we present a short summary and outlook.

\section{THE COMPLETE RQKT TO THE FIRST ORDER IN $\hbar$}

We start with the Lorentz-covariant and gauge-invariant Wigner function $W(x, p)$ defined as [41]

$$
W_{\alpha \beta}=\int \frac{d^{4} y}{(2 \pi)^{4}} e^{-i p \cdot y}\left\langle\bar{\psi}_{\beta}\left(x_{+}\right) U\left(x_{+}, x_{-}\right) \psi_{\alpha}\left(x_{-}\right)\right\rangle,
$$

where $x_{ \pm}=x \pm y / 2, \alpha$ and $\beta$ are spinor indices and $U\left(x_{+}, x_{-}\right)$is the gauge link. This matrix can be expanded in terms of the 16 independent $\Gamma$-matrices,

$$
W=\frac{1}{4}\left[\mathcal{F}+i \gamma^{5} \mathcal{P}+\gamma^{\mu} \mathcal{V}_{\mu}+\gamma^{5} \gamma^{\mu} \mathcal{A}_{\mu}+\frac{1}{2} \sigma^{\mu \nu} \mathcal{S}_{\mu \nu}\right],
$$

where $\mathcal{F}, \mathcal{P}, \mathcal{V}_{\mu}, \mathcal{A}_{\mu}$ and $\mathcal{S}_{\mu \nu}$ are all real functions of $x$ and $p$ representing the scalar, pseudoscalar, vector, axial-vector and antisymmetric tensor components, respectively. These 16 Wigner functions satisfy 32 closed Wigner equations derived from the Dirac equation [41]. Under the external or self-consistent mean field approximation, and to the first order in $\hbar$, they are given by,

$$
\begin{gathered}
p^{\mu} \mathscr{V}_{\mu}=m \mathscr{F}, \\
-\frac{1}{2} \hbar \nabla^{\mu} \mathscr{A}_{\mu}=m \mathscr{P} \\
p_{\mu} \mathscr{F}+\frac{1}{2} \hbar \nabla^{\nu} \mathcal{S}_{\mu \nu}=m \mathscr{V}_{\mu}, \\
\frac{1}{2} \hbar \nabla_{\mu} \mathscr{P}-\frac{1}{2} \epsilon_{\mu \nu \rho \sigma} p^{\nu} \mathcal{S}^{\rho \sigma}=m \mathscr{A}_{\mu}, \\
\frac{1}{2} \hbar\left(\nabla_{\mu} \mathscr{V}_{\nu}-\nabla_{\nu} \mathscr{V}_{\mu}\right)-\epsilon_{\mu \nu \rho \sigma} p^{\rho} \mathscr{A}^{\sigma}=m \mathscr{A}_{\mu \nu},
\end{gathered}
$$

together with those obtained from the imaginary part of the original Wigner equation,

$$
\begin{gathered}
\nabla^{\mu} \mathscr{V}_{\mu}=0, \\
p^{\mu} \mathscr{A}_{\mu}=0, \\
\frac{1}{2} \hbar \nabla_{\mu} \mathscr{F}-p^{\nu} \mathcal{S}_{\mu \nu}=0, \\
p_{\mu} \mathscr{P}+\frac{1}{4} \hbar \epsilon_{\mu \nu \rho \sigma} \nabla^{\nu} \mathcal{S}^{\rho \sigma}=0, \\
\left(p_{\mu} \mathscr{V}_{\nu}-p_{\nu} \mathscr{V}_{\mu}\right)+\frac{1}{2} \hbar \epsilon_{\mu \nu \rho \sigma} \nabla^{\rho} \mathscr{A}^{\sigma}=0
\end{gathered}
$$

where $\nabla^{\mu}=\partial_{x}^{\mu}-F^{\mu \nu} \partial_{\nu}^{p}, \epsilon_{\mu \nu \rho \sigma}$ is the completely antisymmetric tensor with the convention $\epsilon^{0123}=1$, and the electric charge $e$ is included in the gauge field tensor $F^{\mu \nu}$ for brevity.

Here, we note in particular that Eqs. (3)-(12) are obtained from Wigner equations by expanding the coefficients in terms of $\hbar^{n}$ and keeping only terms up to $O(\hbar)$. They are valid definitely up to the order of $O(\hbar)$. There are however two exceptions, i.e., Eqs. (4) and (11) are valid up to $O\left(\hbar^{2}\right)$. This is important when we drive the transport equations and some of the quantum effects up to $O(\hbar)$ and we will come to this point later in the following of this paper.

It turns out that we can choose the scalar $\mathscr{F}$, corresponding to the mass density, and the axial vector $\mathscr{A}_{\mu}$, corresponding to the SPV, as the independent fundamental components and sort the 32 Wigner equations in a concise form as follows.

(i) Eleven of them provide the explicit expressions of other components, $\mathscr{P}, \mathscr{V}_{\mu}$ and $\mathcal{S}_{\mu \nu}$, in terms of $\mathscr{F}$ and $\mathscr{A}_{\mu}$ as given by

$$
\begin{gathered}
\mathscr{P}=-\frac{\hbar}{2 m} \nabla^{\mu} \mathscr{A}_{\mu}, \\
\mathscr{V}_{\mu}=\frac{1}{m} p_{\mu} \mathscr{F}-\frac{\hbar}{2 m^{2}} \epsilon_{\mu \nu \rho \sigma} \nabla^{\nu} p^{\rho} \mathscr{A}^{\sigma}, \\
\mathcal{S}_{\mu \nu}=-\frac{1}{m} \epsilon_{\mu \nu \rho \sigma} p^{\rho} \mathscr{A}^{\sigma}+\frac{\hbar}{2 m^{2}}\left(\nabla_{\mu} p_{\nu} \mathscr{F}-\nabla_{\nu} p_{\mu} \mathscr{F}\right) .
\end{gathered}
$$

Here, we note that Eq. (13) is just obtained from Eq. (4) and is valid up to $O\left(\hbar^{2}\right)$. The expressions of $\mathscr{V}_{\mu}$ and $\mathcal{S}_{\mu \nu}$ in terms of $\mathscr{F}$ and $\mathscr{A}_{\mu}$ given by Eqs. (14) and (15) are obtained by solving Eqs. (7) and (5).

(ii) Five of them provide the modified on-shell conditions for $\mathscr{F}$ and $\mathscr{A}_{\mu}$. They are given by, 


$$
\begin{aligned}
& \left(p^{2}-m^{2}\right) \mathscr{F}=-\frac{\hbar}{m} p^{\mu} \tilde{F}_{\mu \nu} \mathscr{A}^{\nu}, \\
& \left(p^{2}-m^{2}\right) \mathscr{A}_{\mu}=-\frac{\hbar}{m} p^{\nu} \tilde{F}_{\mu \nu} \mathscr{F},
\end{aligned}
$$

where $\tilde{F}_{\mu \nu}=\epsilon_{\mu \nu \rho \sigma} F^{\rho \sigma} / 2$ is the dual field strength tensor. They are obtained by inserting Eq. (14) into (3), Eqs. (13) and (15) into (6) respectively.

(iii) Another five lead to the transport equations for $\mathscr{F}$ and $\mathscr{A}_{\mu}$ respectively. They are given by

$$
\begin{gathered}
p \cdot \nabla \mathscr{F}=\frac{\hbar}{2 m} p^{\mu}\left(\partial_{\lambda}^{x} \tilde{\mathcal{F}}_{\mu \nu}\right) \partial_{p}^{\lambda} \mathscr{A}^{\nu} \\
p \cdot \nabla \mathscr{A}_{\mu}=F_{\mu \nu} \mathscr{A}^{\nu}+\frac{\hbar}{2 m} p^{\nu}\left(\partial_{\lambda}^{x} \tilde{\mathcal{F}}_{\mu \nu}\right) \partial_{p}^{\lambda} \mathscr{F},
\end{gathered}
$$

and are obtained by inserting Eq. (14) into Eq. (8), Eqs. (13) and (15) into Eq. (11) respectively. Here, it is important to note that, by inserting Eqs. (13) and (15) into Eq. (11) to obtain the transport equation for $\mathscr{A}_{\mu}$, we find no contribution at the zeroth order in $\hbar^{n}$ at both sides of the equation. The intermediate equations obtained in the derivation is for $\hbar p$. $\nabla \mathscr{A}_{\mu}$ rather for $p \cdot \nabla \mathscr{A}_{\mu}$ directly. We need to keep the contributions up to the second order in the intermediate equations in order to get the first order contribution to $p \cdot \nabla \mathscr{A}_{\mu}$. It is fortunate that, as mentioned earlier, Eq. (11) is still valid at the second order. After canceling an overall factor $\hbar$ at both sides, we arrive at the transport equation for $\mathscr{A}_{\mu}$ up to the first order as given by Eq. (19).

(iv) There is another equation, i.e., Eq. (9), provides a subsidiary condition for the SPV, i.e.,

$$
p \cdot \mathscr{A}=0
$$

It removes one component of $\mathscr{A}_{\mu}$ from independent dynamical components and reduces the number of independent components to 4 .

(v) Finally, it is remarkable that to the first order in $\hbar$, all the rest 10 of the 32 Wigner equations, i.e., Eqs. (10) and (12) are not independent. They are satisfied automatically provided that Eqs. (13)-(20) given above are fulfilled. Hence we do not need to consider them up to the first order in $\hbar$.

At the end of the section, we would like to emphasize once more that although many of the results presented in this section are just a review and a resorting of the results presented in literature such as those in Ref. [41], the transport equations given by Eqs. (18) and (19) are different from those given in literature where only the leading order contribution to $p \cdot \nabla \mathscr{A}_{\mu}$ was included. We suggest that Eqs. (18) and (19) are regarded as the first order transport equations because they provide the first order solution of $\mathscr{F}$ and $\mathscr{A}_{\mu}$ although they are derived by keeping the second contributions in the intermediate equations. We see that explicit first order terms in Eqs. (18) and (19). They form the basis for the upcoming studies of spin effects presented in the following of this paper.

\section{THE TRANSPORT EQUATIONS AT $O(\hbar)$}

In Sec. II, we have just shown that, by choosing $\mathscr{F}$ and $\mathscr{A}_{\mu}$ as independent components, to the first order in $\hbar$, the 32 Wigner equations are replaced by 5 on-shell conditions given by Eqs. (16) and (17), and 5 transport equations given by Eqs. (18) and (19) for $\mathscr{F}$ and $\mathscr{A}$, and a subsidiary condition given by Eq. (20) for $\mathscr{A}$. The other 11 Wigner functions, $\mathscr{P}, \mathscr{V}_{\mu}$ and $\mathcal{S}_{\mu \nu}$, are expressed in terms of $\mathscr{F}$ and $\mathscr{A}_{\mu}$ explicitly by Eqs. (13)-(15). In this section, we present the general solution of the on-shell conditions and present the transport equations in different forms.

The general solution of the modified on-shell conditions given by Eqs. (16) and (17) is given by

$$
\begin{aligned}
& \mathscr{F}=\delta\left(p^{2}-m^{2}\right) \mathcal{F}+\frac{\hbar}{m} \tilde{F}_{\mu \nu} p^{\mu} \mathcal{A}^{\nu} \delta^{\prime}\left(p^{2}-m^{2}\right), \\
& \mathscr{A}_{\mu}=\delta\left(p^{2}-m^{2}\right) \mathcal{A}_{\mu}+\frac{\hbar}{m} p^{\nu} \tilde{F}_{\mu \nu} \mathcal{F} \delta^{\prime}\left(p^{2}-m^{2}\right),
\end{aligned}
$$

where $\mathcal{F}$ and $\mathcal{A}_{\mu}$ are arbitrary functions of $x$ and $p$ that are nonsingular at $p^{2}=m^{2}$. They are determined by the transport equations given by Eqs. (18) and (19). Here, we would like to note that one can just fix $p_{0}$ in $\mathcal{F}$ and $\mathcal{A}_{\mu}$ at $p_{0}=E_{p} \equiv \sqrt{\mathbf{p}^{2}+m^{2}}$ (for positive energy solution, and $p_{0}=-E_{p}$ for negative energy solution) due to on-shell conditions in Eqs. (16) and (17). If we would choose not to fix it but take it as an independent variable, the term with $\delta^{\prime}\left(p^{2}-m^{2}\right)$ will contribute back to the $\delta\left(p^{2}-m^{2}\right)$ term after a partial integration. The difference between them vanishes by a redefinition of $\mathcal{F}$ and $\mathcal{A}_{\mu}$ in Eqs. (21) and (22).

In practice, it is more convenient to take $\mathcal{F}$ and $\mathcal{A}_{\mu}$ as independent fundamental quantities instead of $\mathscr{F}$ and $\mathscr{A}_{\mu}$. This is particularly the case when a transient electromagnetic (EM) field or a scattering process is considered. In this case, there is no EM field or interaction in the far past or the far future. As can be seen from Eqs. (21) and (22), both $\mathscr{F}$ and $\mathcal{A}_{\mu}$ contribute to $\mathscr{F}$ or $\mathscr{A}_{\mu}$ if the interaction exists. In the far past or the far future, $\tilde{F}_{\mu \nu}=0$ so that $\mathcal{F}$ and $\mathcal{A}_{\mu}$ contribute only to $\mathscr{F}$ and $\mathscr{A}_{\mu}$ separately.

By substituting Eqs. (21) and (22) into Eqs. (18) and (19), we obtain the transport equations for $\mathcal{F}$ and $\mathcal{A}_{\mu}$ in the Lorentz covariant form, 


$$
\begin{aligned}
p \cdot \nabla\left[\mathcal{F} \delta\left(p^{2}-m^{2}\right)+\frac{\hbar}{m} \tilde{F}_{\mu \nu} p^{\mu} \mathcal{A}^{\nu} \delta^{\prime}\left(p^{2}-m^{2}\right)\right] \\
=\frac{\hbar}{2 m}\left(\partial_{\lambda}^{x} \tilde{F}_{\mu \nu}\right) \partial_{p}^{\lambda}\left[p^{\mu} \mathcal{A}^{\nu} \delta\left(p^{2}-m^{2}\right)\right], \\
p \cdot \nabla\left[\mathcal{A}_{\mu} \delta\left(p^{2}-m^{2}\right)+\frac{\hbar}{m} p^{\nu} \tilde{F}_{\mu \nu} \mathcal{F} \delta^{\prime}\left(p^{2}-m^{2}\right)\right] \\
=F_{\mu \nu}\left[\mathcal{A}^{\nu} \delta\left(p^{2}-m^{2}\right)+\frac{\hbar}{m} p_{\lambda} \tilde{F}^{\nu \lambda} \mathcal{F} \delta^{\prime}\left(p^{2}-m^{2}\right)\right] \\
\quad+\frac{\hbar}{2 m}\left(\partial_{\lambda}^{x} \tilde{F}_{\mu \nu}\right) \partial_{p}^{\lambda}\left[p^{\nu} \mathcal{F} \delta\left(p^{2}-m^{2}\right)\right]
\end{aligned}
$$

together with the subsidiary condition,

$$
p \cdot \mathcal{A} \delta\left(p^{2}-m^{2}\right)=0
$$

We see clearly that, by choosing $\mathcal{F}$ and $\mathcal{A}_{\mu}$ as independent fundamental quantities, we are left only with transport equations, Eqs. (23) and (24), and the subsidiary condition given by Eq. (25). All the 16 Wigner functions can be expressed explicitly in terms of $\mathcal{F}$ and $\mathcal{A}_{\mu}$.

Due to the singular $\delta$ function or initial value problem in covariant Wigner functions [45], Eqs. (23) and (24) are not suitable for numerical evaluations. For this purpose, we carry out the integration over $p_{0}$ to get rid of the singular $\delta$-function. Integrating $p_{0}$ from 0 to $\infty$ picks up the particle's contributions while $-\infty$ to 0 for antiparticles. For brevity, we present only results for the particle's contributions in the following. The antiparticle's contribution is similar. By doing this, we obtain the integrated transport equations and the subsidiary condition as

$$
\begin{gathered}
p \cdot \nabla \mathcal{F}=-\frac{\hbar p^{\mu}}{2 m}\left[\frac{\tilde{F}_{\mu \nu} \bar{p}^{\lambda} \nabla_{\lambda}}{E_{p}^{2}}-\left(\bar{\partial}_{x}^{\lambda} \tilde{F}_{\mu \nu}\right) \bar{\partial}_{\lambda}^{p}\right] \mathcal{A}^{\nu}, \\
p \cdot \nabla \mathcal{A}_{\mu}=F_{\mu \nu} \mathcal{A}^{\nu}-\frac{\hbar p^{\nu}}{2 m}\left[\frac{\tilde{F}_{\mu \nu} \bar{p}^{\lambda} \nabla_{\lambda}}{E_{p}^{2}}-\left(\bar{\partial}_{x}^{\lambda} \tilde{F}_{\mu \nu}\right) \bar{\partial}_{\lambda}^{p}\right] \mathcal{F}, \\
p \cdot \mathcal{A}=0,
\end{gathered}
$$

where $p$ is now on the mass-shell, i.e., $p_{0}=E_{p}$, the derivative with respect to $p_{0}$ that was contained in the operator $\nabla^{\mu}$ is removed, $\bar{p}^{\mu}=p^{\mu}-(n \cdot p) n^{\mu}=(0, \mathbf{p})$, $\bar{\partial}_{\mu}=\partial_{\mu}-(n \cdot \partial) n_{\mu}=(0, \partial)$ and $n=(1, \mathbf{0})$. To arrive at the results given by Eqs. (26)-(28), we have taken $p_{0}$ in $\mathcal{F}$ and $\mathcal{A}_{\mu}$ as $p_{0}=E_{p}$ before integrating out $p_{0}$ so that the terms with derivative of $p_{0}$ does not appear.

To do numerical calculations, it is more convenient to rewrite them in the three-dimensional form,

$$
\begin{aligned}
\left(\nabla_{t}\right. & +\mathbf{v} \cdot \boldsymbol{\nabla}) \mathcal{F} \\
= & -\frac{\hbar}{2 m E_{p}}\left[(\mathbf{B}+\mathbf{E} \times \mathbf{v})\left(\mathbf{v} \cdot \boldsymbol{\nabla}+E_{p} \overleftarrow{\nabla}_{x} \cdot \nabla_{p}\right)\right. \\
& \left.-(\mathbf{B} \cdot \mathbf{v})\left(\mathbf{v} \cdot \boldsymbol{\nabla}+E_{p} \overleftarrow{\nabla}_{x} \cdot \nabla_{p}\right) \mathbf{v}\right] \cdot \mathcal{A}, \\
\left(\nabla_{t}\right. & +\mathbf{v} \cdot \boldsymbol{\nabla}) \mathcal{A}+\mathbf{E}(\mathbf{v} \cdot \mathcal{A})-\mathbf{B} \times \mathcal{A} \\
= & -\frac{\hbar}{2 m E_{p}}(\mathbf{B}+\mathbf{E} \times \mathbf{v})\left(\mathbf{v} \cdot \boldsymbol{\nabla}+E_{p} \overleftarrow{\nabla}_{x} \cdot \nabla_{p}\right) \mathcal{F},
\end{aligned}
$$

where $\mathbf{v}=\mathbf{p} / E_{p}, \nabla_{t}=\partial_{t}+\mathbf{E} \cdot \boldsymbol{\nabla}_{p}, \boldsymbol{\nabla}=\boldsymbol{\nabla}_{x}+\mathbf{B} \times \boldsymbol{\nabla}_{p}$ and the left arrow over $\boldsymbol{\nabla}_{x}$ denotes that it acts only on the EM fields on the left. Note that only in the three-dimensional form, we are left with 4 independent distribution functions $\mathcal{F}$ and $\mathcal{A}$ determined by 4 transport equations. The time component $\mathcal{A}^{0}=\mathbf{v} \cdot \mathcal{A}$ is not independent any more.

Now, we have successfully derived the quantum kinetic equations in three different forms: the covariant form [Eqs. (23) and (24)], the integrated form [Eqs. (26) and (27)] and the three-dimensional form [Eqs. (29) and (30)]. They are equivalent to each other and can be used for different purposes. As has been mentioned at the end of Sec. II, we emphasize in particular that the kinetic equations presented above are complete up to the first order of $\hbar$. They are different from those obtained before in literature such as Refs. [39-43] where only the zeroth order of $\mathcal{A}_{\mu}$ was considered.

Before we proceed to use these complete first order equations to study how different spin effects arise from RQKT, for comparison, we present the "simplified version" by keeping only zeroth order of $\mathscr{A}_{\mu}$ in the transport equations at the end of this section.

As we can see from Eq. (14), if we study only quantum effects determined by the vector current $j_{\mu}=\int d^{4} p \mathscr{V}_{\mu}$ and/or the energy momentum tensor $T_{\mu \nu}=\int d^{4} p p_{\nu} \mathscr{V}_{\mu}$ up to the first order in $\hbar$, we need to consider only the zeroth order contribution of $\mathscr{A}_{\mu}$. In this case, the transport equations for $\mathscr{A}_{\mu}$ reduce to those obtained before in e.g., Refs. [39-43]. We now rewrite them in a rather intuitive way. We define

$$
P s_{\mu}=\mathcal{A}_{\mu} / \mathcal{F},
$$

where the unit vector $s$ satisfies $s^{2}=-1$ and $p \cdot s=0$. $P s_{\mu}$ together denotes the polarization vector where $P$ is the magnitude and $s_{\mu}$ is the unit vector. To the zeroth order of $\hbar, P$ and $s_{\mu}$ are decoupled from each other and from $\mathcal{F}$ as well, i.e.,

$$
\begin{gathered}
p \cdot \nabla\left[P \delta\left(p^{2}-m^{2}\right)\right]=0, \\
p \cdot \nabla\left[s_{\mu} \delta\left(p^{2}-m^{2}\right)\right]=F_{\mu \nu} s^{\nu} \delta\left(p^{2}-m^{2}\right) .
\end{gathered}
$$

To the first order in $\hbar$, the equation of motion for $\mathcal{F}$ can be rewritten as 


$$
\begin{aligned}
p & \cdot \nabla\left[\mathcal{F} \delta\left(p^{2}-m^{2}-2 E_{p} \Delta E\right)\right] \\
& =\frac{\hbar}{2 m}\left(\partial_{\lambda}^{x} \tilde{F}^{\rho \sigma}\right) \partial_{p}^{\lambda}\left[p_{\rho} s_{\sigma} P \mathcal{F} \delta\left(p^{2}-m^{2}-2 E_{p} \Delta E\right)\right] .
\end{aligned}
$$

This indicates that all quantum corrections have been absorbed into the effective energy shift due to the interaction between the EM field and spin,

$$
\Delta E=-\hbar P \tilde{F}^{\rho \sigma} p_{\rho} s_{\sigma} / 2 m E_{p} .
$$

We call it the effective energy because the polarization $P$ is an ensemble averaged quantity that is unity for the pure state. We see clearly that, under such approximations, the system remains unpolarized if the initial condition is unpolarized. None of the spin effects such as the chiral anomaly, GPE and CSE can be derived in a self-contained manner from RQKT at this level.

\section{DIFFERENT SPIN EFFECTS}

Using the complete first order results presented in Secs. II and III, we can derive different quantum effects from RQKT. In this section, we present results for different spin effects.

\section{A. The CSE with mass correction}

The axial current can be induced along the external magnetic field imposed on a charged fermion system. This is the so called CSE. With the general solution of $\mathscr{A}_{\mu}$ given by Eq. (22), we immediately obtain CSE with mass correction. We take the global equilibrium solution under constant EM field [46],

$$
\begin{gathered}
\mathcal{A}_{\mu}=0, \\
\mathcal{F}=\frac{m}{2 \pi^{3}}\left[\frac{\theta(u \cdot p)}{e^{(u \cdot p-\mu) / T}+1}+\frac{\theta(-u \cdot p)}{e^{-(u \cdot p-\mu) / T}+1}\right],
\end{gathered}
$$

where $u^{\mu}$ is the fluid velocity and has been identified as $n^{\mu}$ in this calculation. Substituting them into Eq. (22) and integrating over $p$, we obtain

$$
j_{5}^{\mu}=\int d^{4} p \mathscr{A}^{\mu}=\sigma B^{\mu},
$$

where the transport coefficient $\sigma$ is given by

$$
\begin{gathered}
\sigma=\frac{\hbar}{2 \pi^{2}} \int_{0}^{\infty} d p\left(n_{+}-n_{-}\right), \\
n_{ \pm}=\frac{1}{e^{\left(E_{p} \mp \mu\right) / T}+1} .
\end{gathered}
$$

This is exactly the same result as that obtained in Ref. [47], where the Kubo formula and axial anomaly equation were used. At the chiral limit of $m=0$, we obtain the wellknown result

$$
\left.\sigma\right|_{m=0}=\hbar \frac{\mu}{2 \pi^{2}},
$$

and at zero temperature, we obtain

$$
\left.\sigma\right|_{T \rightarrow 0}=\hbar \frac{\mu}{2 \pi^{2}} \sqrt{1-\frac{m^{2}}{\mu^{2}}} .
$$

In the present work, we did not recover the CSE induced by vorticity because we could not determine the possible vorticity contribution in Eq. (36) from Eqs. (23) and (24) in the absence of collision terms.

\section{B. The chiral anomaly}

Chiral anomaly is a fascinating quantum field effect that is absent at the classical level. A proper RQKT should be able to account for this effect. In the Wigner function formalism presented in Secs. II and III, chiral anomaly can be derived directly from Eq. (13).

By inserting the general solution Eq. (22) of the modified mass-shell condition into Eq. (13) and integrating over the momentum $p$, we obtain,

$$
\hbar \partial_{\mu}^{x} j_{5}^{\mu}=-2 m j_{5}+\hbar^{2} C E \cdot B
$$

where $j_{5}=\int d^{4} p \mathscr{P}$, and the constant $C$ is given by

$$
C=\int d^{4} p \partial_{p}^{\lambda} p_{\lambda} \mathcal{F} \delta^{\prime}\left(p^{2}-m^{2}\right) / m
$$

Here, we have dropped the nonsingular term that vanishes after the integration. From Eq. (43), we see clearly that the chiral anomaly is obtained automatically by putting $m=0$.

From Eq. (43), again, we see that, to obtain the chiral anomaly at the first order in $\hbar$, it is necessary to keep the second order contributions in the intermediate equation because this leads to the results for $\hbar \partial_{\mu}^{x} j_{5}^{\mu}$ rather than $\partial_{\mu}^{x} j_{5}^{\mu}$ directly. Here, we note in particular that the $\hbar$ factor before $\partial_{\mu}^{x} j_{5}^{\mu}$ is expected from dimension counting in the relationship between the pseudoscalar $j_{5}$ and the divergence of the axial-vector $j_{5}^{\mu}$. This seems different from what we see in the literature where natural unit is usually used so that $\hbar$ is omitted. As mentioned earlier, the expression of $\mathscr{P}$ in Eq. (13) is valid up to the second order in $\hbar$, hence we can keep the second order contributions in Eq. (43). We also see from Eq. (13) that the first order correction to $\mathscr{A}^{\mu}$ actually contributes to the second order correction to $\mathscr{P}$ and it is the second order contribution of $\mathscr{P}$ that gives rise to the second term on the rhs of Eq. (43) and leads to the chiral anomaly. The leading contribution to $j_{5}$ is at order of $O(\hbar)$.

At the chiral limit $m=0$, an overall factor $\hbar$ is removed from both sides of Eq. (43), so that the anomaly term $E \cdot B$ actually appears at $O(\hbar)$.

We calculate the constant $C$ by choosing two specific solutions in limiting cases: the free vacuum solution [48] 


$$
\mathcal{A}_{\mu}=0, \quad \mathcal{F}=-m / 4 \pi^{3},
$$

and the global equilibrium solution with Fermi-Dirac distribution given by Eq. (37) in chiral limit of $m=0$. For the free vacuum solution, we can use the regularization

$$
\delta^{\prime}(x)=\frac{1}{\pi} \operatorname{Im} \frac{1}{(x+i \epsilon)^{2}},
$$

where $\epsilon$ is a small positive number and obtain

$$
\begin{aligned}
C & =-\frac{1}{4 \pi^{4}} \operatorname{Im} \int d^{4} p \partial_{p}^{\lambda} \frac{p_{\lambda}}{\left(p^{2}-m^{2}+i \epsilon\right)^{2}} \\
& =-\frac{1}{4 \pi^{4}} \int d^{4} p_{E} \partial_{p_{E}}^{\lambda} \frac{p_{E \lambda}}{\left(p_{E}^{2}+m^{2}\right)^{2}}=-\frac{1}{2 \pi^{2}} .
\end{aligned}
$$

Here, to carry out the integrations, we have used Wick rotation and four-dimensional Gauss theorem. For the global equilibrium solution given by Eq. (37) at chiral limit, we will integrate out the 4-momentum directly instead of by using Wick rotation due to nontrivial analytic structure and obtain

$$
C=-\left.\frac{1}{2 \pi^{2}}\left(n_{+}+n_{-}\right)\right|_{E_{p}=0}=-\frac{1}{2 \pi^{2}} .
$$

It is interesting to note that, although the results of chiral anomaly obtained in the above-mentioned two cases are exactly the same, they originate from different momentum regions. For the free vacuum solution, there is no suppression at large momentum and the finite contribution comes from the large momentum, while for the chiral limit with Fermi-Dirac distribution, there is an exponential suppression at large momentum, the finite contribution is from the infrared region. This indicates again further studying on where chiral anomaly comes from in RQKT $[35,37,44]$ is necessary and important.

\section{The quantum magnetization effect}

The magnetic moment density is determined by the space components of the antisymmetric tensor $\mathcal{S}_{\mu \nu}$ [48]. We still take the global equilibrium solution given by Eqs. (36) and (37) with constant thermal vorticity tensor $\Omega_{\mu \nu}=\partial_{\mu}^{x} \beta_{\nu}-$ $\partial_{\nu}^{x} \beta_{\mu}$, where $\beta_{\mu}=u_{\mu} / T$. We insert it into Eq. (15) to get $\delta_{\mu \nu}$. It follows that the magnetic moment density or magnetization vector in the local co-moving frame is given by

$$
M_{\mu}=\frac{1}{2} \epsilon_{\nu \mu \alpha \beta} u^{\nu} \int d^{4} p \mathcal{S}^{\alpha \beta}=\hbar \kappa B_{\mu}-\hbar \rho \omega_{\mu} / m,
$$

where $B_{\mu}=\tilde{F}_{\mu \nu} u^{\nu}$ is the magnetic field in the fluid rest frame, $\omega_{\mu}=\epsilon_{\mu \nu \alpha \beta} u^{\nu} \partial_{x}^{\alpha} u^{\beta} / 2$ is the vorticity vector, $\kappa$ is the magnetic susceptibility and is given by

$$
\kappa=\frac{m}{2 \pi^{2}} \int_{0}^{\infty} \frac{d p}{E_{p}}\left(n_{+}+n_{-}\right)
$$

and $\rho$ is the charge density

$$
\rho=\frac{1}{\pi^{2}} \int_{0}^{\infty} d p p^{2}\left(n_{+}-n_{-}\right) .
$$

In the zero temperature limit, we obtain the analytic expressions

$$
\begin{gathered}
\left.\kappa\right|_{T \rightarrow 0}=\frac{m}{2 \pi^{2}} \ln \frac{|\mu|+\sqrt{\mu^{2}-m^{2}}}{m}, \\
\left.\rho\right|_{T \rightarrow 0}=\frac{1}{3 \pi^{2}}\left(\mu^{2}-m^{2}\right)^{3 / 2} .
\end{gathered}
$$

Again, we would like to note that we did not include contributions in Eq. (36) due to vorticity.

The presence of $\hbar$ on the rhs shows that magnetization due to magnetic field or vorticity is a pure quantum effect. As we all know, charged vorticity can induce magnetic moment even in classical physics. But the result presented here is different from this classical mechanism and it represents ensemble average of the intrinsic magnetic moment that does not depend on the origin of the coordinate that we choose. The term provides the relationship between the vorticity and the induced spin magnetization. We emphasize that, though obtained with the Fermi-Dirac distribution, the vorticity term in Eq. (49) is valid if $\mathcal{F}$ is chosen as any function of $p \cdot u$.

\section{GPE generation}

Let us consider a fermionic system subject to a transient external EM field. We suppose that at $t=0$, there is no EM field, and the system is unpolarized so that $\mathcal{A}=0$ and $\mathcal{F} \neq 0$. The EM field comes up at the next moment and the evolution of $\mathcal{A}$ after an infinitesimal time interval is determined by

$\nabla_{t} \mathcal{A}=-\frac{\hbar}{2 m E_{p}}(\mathbf{B}+\mathbf{E} \times \mathbf{v})\left(\mathbf{v} \cdot \boldsymbol{\nabla}+E_{p} \overleftarrow{\boldsymbol{\nabla}}_{x} \cdot \boldsymbol{\nabla}_{p}\right) \mathcal{F}$

We see clearly that the polarization is generated along the magnetic field due to the inhomogeneous distribution of $\mathcal{F}$ in phase space.

From Eq. (54), one might also conclude that, if there is no external EM field at $t=0$, the transport equations for $\mathcal{F}$ and $\mathcal{A}$ would decouple from each other so that GPE became impossible from the unpolarized initial state. This is not true in practice since the self-consistent EM field is determined by Maxwell's equations $\partial_{\mu} F^{\mu \nu}=e^{2} j^{\nu}\left(e^{2}\right.$ is due to the extra $e$ in $F^{\mu \nu}$ ) that close with transport equations that in turn determine the current $j_{\mu}$. The relationship between the vorticity and EM field can be seen more 
directly if we rewrite Maxwell's equations as quadratic equations for the field tensor, i.e., [41]

$$
\partial_{\lambda} \partial^{\lambda} F_{\mu \nu}=e^{2}\left(\partial_{\mu} j_{\nu}-\partial_{\nu} j_{\mu}\right)
$$

We see explicitly that it is the vorticity of $j_{\mu}$ instead of the current itself that induces EM field tensor. The induced EM field will couple $\mathcal{A}$ and $\mathcal{F}$ together and generate the polarization along the direction of vorticity. This provides a mechanism to generate the GPE from vorticity in the current. We emphasize that here we have no collision terms but only relativistic quantum effects. Further studies can be carried out numerically for specified systems in future works.

\section{SUMMARY AND OUTLOOK}

By choosing the scalar $\mathscr{F}$ and the axial vector $\mathscr{A}_{\mu}$ as independent components, we have presented the RQKT for particles with spin- $1 / 2$ up to the first order in $\hbar$ derived from the covariant Wigner function formalism completely and in a concise form. This form shows explicitly that the 32 Wigner equations can be replaced by 5 transport equations, 5 modified on-shell conditions and 1 subsidiary constraint. We have solved the modified on-shell conditions and obtained the general solution. Using this general solution, the 32 Wigner equations are in practice replaced by 4 independent transport equations. We have presented these transport equations in three different forms, the covariant, integrated and three-dimensional forms, that can be used for different purposes. We have demonstrated that different spin effects such as the chiral anomaly, CSE, quantum magnetization effect and GPE can arise from the RQKT automatically.

We have pointed out in particular that these spin effects arise only when we consider Wigner equations up to the first order in $\hbar$ completely. None of them arises automatically if we consider only the current and energy momentum tensor to the first order but take the axial vector $\mathscr{A}_{\mu}$ only to the zeroth order.

By calculating the axial current $j_{5 \mu}=\int d^{4} p \mathscr{A}_{\mu}$ and $j_{5}=\int d^{4} p \mathscr{P}$, we have successfully shown that CSE with mass correction and the chiral anomaly are obtained automatically in the self-contained manner and lead to correct forms as obtained in other approaches. We have also calculated the quantum magnetization effect and obtained the simple relationship $M_{\mu}=\hbar \kappa B_{\mu}-\hbar \rho \omega_{\mu} / m$. We have also shown in particular that, although no collision term is considered yet, GPE can be generated from current vorticity due to back reactions of EM fields from Maxwell's equations.

Finally we would like to emphasize that the formalism presented provides a practical theoretical framework in RQKT to study different spin effects in different relativistic quantum systems. It can be extended to higher orders in a straightforward manner and is applicable not only in heavyion physics but also other areas such as astrophysics as well as condensed matter physics where polarization effects are involved in massive fermion systems.

\section{ACKNOWLEDGMENTS}

We thank Q. Wang for helpful discussions. This work was supported in part by the National Natural Science Foundation of China under No. 11890713 and No. 11675092, and the Natural Science Foundation of Shandong Province under No. JQ201601.

Note added.-While this work was being completed, we became aware of the work Ref. [49] which also derives the RQKT from Wigner-function formalism.
[1] L. Adamczyk et al. (STAR Collaboration), Nature (London) 548, 62 (2017).

[2] J. Adam et al. (STAR Collaboration), Phys. Rev. C 98, 014910 (2018).

[3] Z. T. Liang and X. N. Wang, Phys. Rev. Lett. 94, 102301 (2005); 96, 039901(E) (2006).

[4] Z. T. Liang and X. N. Wang, Phys. Lett. B 629, 20 (2005).

[5] B. Betz, M. Gyulassy, and G. Torrieri, Phys. Rev. C 76, 044901 (2007).

[6] F. Becattini, F. Piccinini, and J. Rizzo, Phys. Rev. C 77, 024906 (2008).

[7] J. H. Gao, S. W. Chen, W.t. Deng, Z. T. Liang, Q. Wang, and X. N. Wang, Phys. Rev. C 77, 044902 (2008).

[8] F. Becattini, I. Karpenko, M. Lisa, I. Upsal, and S. Voloshin, Phys. Rev. C 95, 054902 (2017).
[9] F. Becattini and I. Karpenko, Phys. Rev. Lett. 120, 012302 (2018).

[10] F. Becattini, W. Florkowski, and E. Speranza, Phys. Lett. B 789, 419 (2019).

[11] R. h. Fang, L. g. Pang, Q. Wang, and X. n. Wang, Phys. Rev. C 94, 024904 (2016).

[12] L. G. Pang, H. Petersen, Q. Wang, and X. N. Wang, Phys. Rev. Lett. 117, 192301 (2016).

[13] W. Florkowski, B. Friman, A. Jaiswal, and E. Speranza, Phys. Rev. C 97, 041901 (2018).

[14] W. Florkowski, B. Friman, A. Jaiswal, R. Ryblewski, and E. Speranza, Phys. Rev. D 97, 116017 (2018).

[15] W. Florkowski, A. Kumar, and R. Ryblewski, Phys. Rev. C 98, 044906 (2018).

[16] Y. Sun and C. M. Ko, Phys. Rev. C 96, 024906 (2017). 
[17] K. Hattori, M. Hongo, X. G. Huang, M. Matsuo, and H. Taya, Phys. Lett. B 795, 100 (2019).

[18] A. Vilenkin, Phys. Rev. D 22, 3080 (1980).

[19] D. E. Kharzeev, L. D. McLerran, and H. J. Warringa, Nucl. Phys. A803, 227 (2008).

[20] K. Fukushima, D. E. Kharzeev, and H. J. Warringa, Phys. Rev. D 78, 074033 (2008).

[21] A. Vilenkin, Phys. Rev. D 20, 1807 (1979).

[22] J. Erdmenger, M. Haack, M. Kaminski, and A. Yarom, J. High Energy Phys. 01 (2009) 055.

[23] N. Banerjee, J. Bhattacharya, S. Bhattacharyya, S. Dutta, R. Loganayagam, and P. Surowka, J. High Energy Phys. 01 (2011) 094.

[24] D. T. Son and P. Surowka, Phys. Rev. Lett. 103, 191601 (2009).

[25] K. Landsteiner, E. Megias, and F. Pena-Benitez, Phys. Rev. Lett. 107, 021601 (2011).

[26] J. H. Gao, Z. T. Liang, S. Pu, Q. Wang, and X. N. Wang, Phys. Rev. Lett. 109, 232301 (2012).

[27] M. A. Stephanov and Y. Yin, Phys. Rev. Lett. 109, 162001 (2012).

[28] D. T. Son and N. Yamamoto, Phys. Rev. D 87, 085016 (2013).

[29] J. W. Chen, S. Pu, Q. Wang, and X. N. Wang, Phys. Rev. Lett. 110, 262301 (2013).

[30] C. Manuel and J. M. Torres-Rincon, Phys. Rev. D 89, 096002 (2014).

[31] C. Manuel and J. M. Torres-Rincon, Phys. Rev. D 90, 076007 (2014).

[32] J. Y. Chen, D. T. Son, M. A. Stephanov, H. U. Yee, and Y. Yin, Phys. Rev. Lett. 113, 182302 (2014).

[33] J. Y. Chen, D. T. Son, and M. A. Stephanov, Phys. Rev. Lett. 115, 021601 (2015).
[34] Y. Hidaka, S. Pu, and D. L. Yang, Phys. Rev. D 95, 091901 (2017).

[35] N. Mueller and R. Venugopalan, Phys. Rev. D 97, 051901 (2018).

[36] A. Huang, S. Shi, Y. Jiang, J. Liao, and P. Zhuang, Phys. Rev. D 98, 036010 (2018).

[37] J. H. Gao, Z. T. Liang, Q. Wang, and X. N. Wang, Phys. Rev. D 98, 036019 (2018).

[38] Y. C. Liu, L. L. Gao, K. Mameda, and X. G. Huang, Phys. Rev. D 99, 085014 (2019).

[39] U. W. Heinz, Phys. Rev. Lett. 51, 351 (1983).

[40] H. T. Elze, M. Gyulassy, and D. Vasak, Nucl. Phys. B276, 706 (1986).

[41] D. Vasak, M. Gyulassy, and H. T. Elze, Ann. Phys. (N.Y.) 173, 462 (1987).

[42] P. Zhuang and U. W. Heinz, Ann. Phys. (N.Y.) 245, 311 (1996).

[43] J. P. Blaizot and E. Iancu, Phys. Rep. 359, 355 (2002).

[44] N. Mueller and R. Venugopalan, Phys. Rev. D 99, 056003 (2019).

[45] S. Ochs and U.W. Heinz, Ann. Phys. (N.Y.) 266, 351 (1998).

[46] In principle, these equilibrium solutions should be derived from kinetic theory. However, since the present formalism lacks the collision term, it is impossible to determine precisely these equilibrium solutions. We will use these well-known solutions as input instead of deriving them.

[47] S. Lin and L. Yang, Phys. Rev. D 98, 114022 (2018).

[48] I. Bialynicki-Birula, P. Gornicki, and J. Rafelski, Phys. Rev. D 44, 1825 (1991).

[49] N. Weickgenannt, X. L. Sheng, E. Speranza, Q. Wang, and D. H. Rischke, arXiv:1902.06513. 